\title{
Fourth-Order Accurate Alternating Group Schemes for the Diffusion Problem
}

\author{
Fuli Qu ${ }^{1}$ and Wenqia Wang ${ }^{2}$ \\ ${ }^{1}$ Basic Courses' Department, Shandong Women's University, Jinan 250300, \\ Shandong, China \\ ${ }^{2}$ School of Mathematics, Shandong University, Jinan 250100, Shandong, China \\ 'qufuli23@163.com, ${ }^{2}$ wangwq@sdu.edu.cn
}

\begin{abstract}
Based on the alternating group strategies, a new high accurate Alternating Group 8point scheme $(A G-8 p)$ for the diffusion problem is presented. The scheme is proved to be unconditionally stable. In numerical experiments, we compare the $A G-8 p$ scheme with the $A G E$ and the ASC-N schemes. Finally, numerical results show that the AG-8p scheme has high accuracy.
\end{abstract}

Keywords: Diffusion problem, Alternating group schemes, Fourth order accuracy, Parallel computing

\section{Introduction}

In many fields of natural science, many phenomena are described by diffusion equation, such as chemical diffusion, biochemistry and certain biological process etc. So the solution of the diffusion equation is one of the most classic problem in the numerical approximation of partial differential equations. Among modern numerical methods, the finite difference method is the most classic one. With the parallel computer's birth and development, some disadvantages appear in different means. For example, explicit scheme is easy to implement and suit for parallel computing, but for stability constraints, it requires small time steps. Implicit scheme is absolutely stable, but for computing slowly unsteady problems, it needs to march with large time steps.

For numerically solving heat equations on parallel computers, two kinds of finite difference methods are constructed. One is the alternating method (see [1-10]). The method uses alternately the explicit scheme and implicit scheme in the time and space direction, which can implement the parallel computation and is unconditionally stable; the other is the domain decomposition method (see [11-12]). In the further research of the alternating method, D. J. Evans introduces the Alternating Group Explicit (AGE) method in [1-2] for solving the parabolic equation. Based on the works of Evans, B. L. Zhang develops the Alternating Segment Explicit-Implicit (ASEI) method and the Alternating Segment Crank-Nicolson (ASCN) method ([3-4]). After that the study of the AGE scheme has been introduced into solving the hyperbolic equation and the dispersive equation respectively in [4] and [9]. All these methods are all unconditionally stable, and in space direction, their truncation errors are all nearly second order. Recently, S. Zhu develops a higher order truncation error approximately, which results from the use of asymmetric three-level approximations ([13]).

In this paper we design a new alternating group scheme with the truncation error of higher order by using asymmetric two-level approximations for the following diffusion problem with periodic solution. The scheme has fourth order accuracy, being unconditionally stable and naturally parallel. 


\section{The Finite Difference Approximation}

We consider the following diffusion problem,

$$
L u=\frac{\partial u}{\partial t}-\frac{\partial^{2} u}{\partial x^{2}}=0, \quad 0<x<l, \quad 0<t<T,
$$

with the initial condition $u(x, 0)=f(x), 0<x<l$ and the boundary condition $u(x, t)=u(x+l, t), 0<t<T$, where $l$ is the constant period.

Let $h$ and $\tau$ be the spatial step size and the time step size respectively. We use $u(x, t)$ to represent the exact solution of Eq. (1) and $U_{i}^{n}$ to denote the numerical approximation of $u\left(x_{i}, t_{n}\right)=u_{i}^{n}$, where $x_{i}=(i-1) h, i=1,2, \cdots, J+1, t_{n}=n \tau, n=0,1, \cdots,[T / \tau]$. In particular, $h=\frac{l}{J}$ for some positive integer $J=8 m$.

In order to increase the order of error in spatial, we introduce the following fourthorder difference scheme

$$
\begin{aligned}
\left(\frac{\partial^{2} u}{\partial x^{2}}\right)_{i}^{n+\frac{1}{2}}= & \frac{2}{3}\left[\frac{u_{i+1}^{n+1}-2 u_{i}^{n+1}+u_{i-1}^{n+1}}{h^{2}}+\frac{u_{i+1}^{n}-2 u_{i}^{n}+u_{i-1}^{n}}{h^{2}}\right]- \\
& \frac{1}{6}\left[\frac{u_{i+2}^{n+1}-2 u_{i}^{n+1}+u_{i-2}^{n+1}}{4 h^{2}}+\frac{u_{i+2}^{n}-2 u_{i}^{n}+u_{i-2}^{n}}{4 h^{2}}\right]+O\left(\tau^{2}+h^{4}\right)
\end{aligned}
$$

On the basis of the above formula, and combining with $\left(\frac{\partial u}{\partial t}\right)_{i}^{n+\frac{1}{2}}=\frac{u_{i}^{n+1}-u_{i}^{n}}{\tau}+O\left(\tau^{2}\right)$, we can get the following eight basic schemes approximating Eq. (1) :

$$
\begin{aligned}
A_{1}:(1+15 r) U_{i}^{n+1} & -16 r U_{i+1}^{n+1}+r U_{i+2}^{n+1} \\
= & -2 r U_{i-2}^{n}+32 r U_{i-1}^{n}+(1-45 r) U_{i}^{n}+16 r U_{i+1}^{n}-r U_{i+2}^{n} \\
A_{2}:-16 r U_{i-1}^{n+1}+ & (1+31 r) U_{i}^{n+1}-16 r U_{i+1}^{n+1}+r U_{i+2}^{n+1} \\
= & -2 r U_{i-2}^{n}+16 r U_{i-1}^{n}+(1-29 r) U_{i}^{n}+16 r U_{i+1}^{n}-r U_{i+2}^{n}
\end{aligned}
$$

$A_{3}: \quad r U_{i-2}^{n+1}-16 r U_{i-1}^{n+1}+(1+29 r) U_{i}^{n+1}-16 r U_{i+1}^{n+1}+2 r U_{i+2}^{n+1}$

$$
=-r U_{i-2}^{n}+16 r U_{i-1}^{n}+(1-31 r) U_{i}^{n}+16 r U_{i+1}^{n}
$$

$A_{4}: \quad r U_{i-2}^{n+1}-16 r U_{i-1}^{n+1}+(1+45 r) U_{i}^{n+1}-32 r U_{i+1}^{n+1}+2 r U_{i+2}^{n+1}$

$$
=-r U_{i-2}^{n}+16 r U_{i-1}^{n}+(1-15 r) U_{i}^{n}
$$

$$
\begin{gathered}
A_{5}: 2 r U_{i-2}^{n+1}-32 r U_{i-1}^{n+1}+(1+45 r) U_{i}^{n+1}-16 r U_{i+1}^{n+1}+r U_{i+2}^{n+1} \\
=(1-15 r) U_{i}^{n}+16 r U_{i+1}^{n}-r U_{i+2}^{n}
\end{gathered}
$$

$$
A_{6}: 2 r U_{i-2}^{n+1}-16 r U_{i-1}^{n+1}+(1+29 r) U_{i}^{n+1}-16 r U_{i+1}^{n+1}+r U_{i+2}^{n+1}
$$

$$
=16 r U_{i-1}^{n}+(1-31 r) U_{i}^{n}+16 r U_{i+1}^{n}-r U_{i+2}^{n}
$$

$A_{7}: r U_{i-2}^{n+1}-16 r U_{i-1}^{n+1}+(1+31 r) U_{i}^{n+1}-16 r U_{i+1}^{n+1}$

$$
=-r U_{i-2}^{n}+16 r U_{i-1}^{n}+(1-29 r) U_{i}^{n}+16 r U_{i+1}^{n}-2 r U_{i+2}^{n}
$$

$A_{8}: r U_{i-2}^{n+1}-16 r U_{i-1}^{n+1}+(1+15 r) U_{i}^{n+1}$

$$
=-r U_{i-2}^{n}+16 r U_{i-1}^{n}+(1-45 r) U_{i}^{n}+32 r U_{i+1}^{n}-2 r U_{i+2}^{n}
$$

where $r=\frac{\tau}{24 h^{2}}$. 


\section{The New Alternating Group 8-point (AG-8p) Scheme}

We assume that $J=8 m$, we provide the implementation of the AG 8-p algorithm for solving the diffusion problem as follows:

I) At the odd time levels, grid nodes can be divided into $m$ groups and use the schemes $A_{1} \sim A_{8}$ for each group from the first node to the last node respectively.

II) At the even time levels, the nodes include a left 4-point group, $m-1$ groups of eight points and a right 4-point group; and we use the schemes $A_{5} \sim A_{8}$ for the left group and the schemes $A_{1} \sim A_{4}$ for the right group. The other $m-1$ groups can be solved by the schemes $A_{1} \sim A_{8}$. The solving scheme is constructed. Such arrangement results in the following linear system.

When $n$ is even number, for solving the $n+1$ time level for $\left(x_{j}, t_{n+1}\right)$, $j=i+1, \cdots, i+8 ; i=8 k, k=0,1, \cdots, m-1$, we use the schemes $A_{1} \sim A_{8}$, and we rewrite the formulae in the following matrix form:

$$
\left(I+r P_{k}\right) \bar{U}^{n+1}=B
$$

where

$$
\bar{U}^{n+1}=\left(U_{i+1}^{n+1}, U_{i+2}^{n+1}, U_{i+3}^{n+1}, U_{i+4}^{n+1}, U_{i+5}^{n+1}, U_{i+6}^{n+1}, U_{i+7}^{n+1}, U_{i+8}^{n+1}\right)^{T},
$$

$P_{k}$ is a non-negative definite symmetric matrix as follows:

$$
P_{k}=\left[\begin{array}{ccccccccc}
15 & -16 & 1 & & \vdots & & & & \\
-16 & 31 & -16 & 1 & \vdots & & & & \\
1 & -16 & 29 & -16 & \vdots & 2 & & & \\
& 1 & -16 & 45 & \vdots & -32 & 2 & & \\
\cdots & \cdots & \ldots & \ldots & \vdots & \ldots & \ldots & \ldots & \ldots \\
& & 2 & -32 & \vdots & 45 & -16 & 1 & \\
& & & 2 & \vdots & -16 & 29 & -16 & 1 \\
& & & & \vdots & 1 & -16 & 31 & -16 \\
& & & & \vdots & & 1 & -16 & 15
\end{array}\right]=\left[\begin{array}{cc}
E_{k} & F_{k} \\
F_{k}^{T} & H_{k}
\end{array}\right],
$$




$$
\begin{gathered}
B=\left(I-r Q_{k}\right) \bar{U}^{n}+b, \\
Q_{k}=\left[\begin{array}{cc}
H_{k} & 0 \\
0 & E_{k}
\end{array}\right], \\
b=\left(-2 r U_{i-1}^{n}+32 r U_{i}^{n},-2 r U_{i}^{n}, 0,0,0,0,-2 r U_{i+9}^{n}, 32 r U_{i+9}^{n}-2 r U_{i+10}^{n}\right)^{T} .
\end{gathered}
$$

Because of $J=8 m, m$ is a positive integer. So we divide the mesh points $x_{1}, x_{2}, \cdots, x_{J}$ which are located in one period into $m$ groups. Later we propose the AG$8 \mathrm{p}$ method by using the formulae $A_{1} \sim A_{8}$ for the eight points respectively in every group. Let $U^{n}=\left(U_{1}^{n}, U_{2}^{n}, \cdots, U_{J}^{n}\right)^{T}$, considering the periodic property of boundary condition, we can obtain:

$$
\left(I+r G_{1}\right) U^{n+1}=\left(I-r G_{2}\right) U^{n},
$$

where

$$
G_{1}=\left[\begin{array}{cccc}
P_{k} & & & \\
& P_{k} & & \\
& & \ddots & \\
& & & P_{k}
\end{array}\right]_{J \times J}, G_{2}=\left[\begin{array}{ccccc}
E_{k} & & & & F_{k}^{T} \\
& P_{k} & & & \\
& & \ddots & & \\
& & & P_{k} & \\
F_{k} & & & & H_{k}
\end{array}\right]_{J \times J},
$$

But for the $n+2$ time level, we can obtain the matrix equation as follows by the same processing method:

$$
\left(I+r G_{2}\right) U^{n+2}=\left(I-r G_{1}\right) U^{n+1},
$$

So we get the complete scheme as follows, namely the Alternating Group 8-points scheme(AG-8p).

$$
\begin{gathered}
B_{1}:\left(I+r G_{1}\right) U^{n+1}=\left(I-r G_{2}\right) U^{n}, \\
B_{2}:\left(I+r G_{2}\right) U^{n+2}=\left(I-r G_{1}\right) U^{n+1}, \\
n=0,2,4, \cdots
\end{gathered}
$$

\section{Stability and Truncation Error Analyses}

With the lemma of Kellogg (see [14]), we can give the stability analysis of the presented algorithm, that is:

Now eliminating $U^{n+1}$ from $B_{1}$ and $B_{2}$ gives:

where

$$
U^{2 n}=T U^{2 n-2}=\cdots=T^{n} U^{0},
$$

$$
T=\left(I+r G_{2}\right)^{-1}\left(I-r G_{1}\right)\left(I+r G_{1}\right)^{-1}\left(I-r G_{2}\right),
$$

Since $G_{1}, G_{2}$ are all non-negative definite, then the following inequalities are derived for any positive integer $n$ and positive $r$

$$
\begin{aligned}
& \quad\left\|T^{n}\right\| \leq\left\|\left(I+r G_{2}\right)^{-1}\right\|_{2}\left\|\left(I-r G_{1}\right)\left(I+r G_{1}\right)^{-1}\right\|_{2}\left\|\left(I-r G_{2}\right)\left(I+r G_{2}\right)^{-1}\right\|_{2} \\
& \cdots\left\|\left(I-r G_{1}\right)\left(I+r G_{1}\right)^{-1}\right\|_{2}\left\|\left(I-r G_{2}\right)\right\|_{2} \\
& \leq\left\|\left(I-r G_{2}\right)\right\|_{2} \leq 1+4 r_{0}, \quad\left(r_{0}=\frac{\tau}{h^{2}}\right)
\end{aligned}
$$

So we prove the AG- $8 \mathrm{p}$ scheme is unconditional stable. Then we have the next theorem. 
Theorem Assume that $n$ is an even number, $r$ is any positive real number, the AG- $8 \mathrm{p}$ method $B_{1} \sim B_{2}$ is unconditionally stable.

We perform the truncation error analysis for the AG- $8 \mathrm{p}$ scheme. The actual computation involves four pairs of schemes which are alternately used between two time levels. These pairs are: $A_{1}$ and $A_{5}, A_{2}$ and $A_{6}, A_{3}$ and $A_{7}, A_{4}$ and $A_{8}$. In fact, we also may regard the AG-8p schemes $B_{1} \sim B_{2}$ as the schemes of three layers, i.e., formulae $A_{1}$ and $A_{5}$ have been combined into the formulae $C_{1}$ and $C_{5}$ as follows, similarly, formulae $A_{2}$ and $A_{6}$ into $C_{2}$ and $C_{6}$, formulae $A_{3}$ and $A_{7}$ into $C_{3}$ and $C_{7}$, formulae $A_{4}$ and $A_{8}$ into $C_{4}$ and $C_{8}$.

$$
\begin{aligned}
& C_{1}:(1+15 r) U_{i}^{n+2}-16 r U_{i+1}^{n+2}+r U_{i+2}^{n+2} \\
& =-4 r U_{i-2}^{n+1}+64 r U_{i-1}^{n+1}-90 r U_{i}^{n+1}+32 r U_{i+1}^{n+1} \\
& -2 r U_{i+2}^{n+1}+(1-15 r) U_{i}^{n}+16 r U_{i+1}^{n}-r U_{i+2}^{n} \\
& C_{2}:-16 r U_{i-1}^{n+2}+(1+31 r) U_{i}^{n+2}-16 r U_{i+1}^{n+2}+r U_{i+2}^{n+2} \\
& =-4 r U_{i-2}^{n+1}+32 r U_{i-1}^{n+1}-58 r U_{i}^{n+1}+32 r U_{i+1}^{n+1}-2 r U_{i+2}^{n+1} \\
& +16 r U_{i-1}^{n}+(1-31 r) U_{i}^{n}+16 r U_{i+1}^{n}-r U_{i+2}^{n} \\
& C_{3}: r U_{i-2}^{n+2}-16 r U_{i-1}^{n+2}+(1+29 r) U_{i}^{n+2}-16 r U_{i+1}^{n+2}+2 r U_{i+2}^{n+2} \\
& =-2 r U_{i-2}^{n+1}+32 r U_{i-1}^{n+1}-62 r U_{i}^{n+1}+32 r U_{i+1}^{n+1}-r U_{i-2}^{n} \\
& +16 r U_{i-1}^{n}+(1-29 r) U_{i}^{n}+16 r U_{i+1}^{n}-2 r U_{i+2}^{n} \\
& C_{4}: r U_{i-2}^{n+2}-16 r U_{i-1}^{n+2}+(1+45 r) U_{i}^{n+2}-32 r U_{i+1}^{n+2}+2 r U_{i+2}^{n+2} \\
& =-2 r U_{i-2}^{n+1}+32 r U_{i-1}^{n+1}-30 r U_{i}^{n+1}-r U_{i-2}^{n} \\
& +16 r U_{i-1}^{n}+(1-45 r) U_{i}^{n}+32 r U_{i+1}^{n}-2 r U_{i+2}^{n} \\
& C_{5}: 2 r U_{i-2}^{n+2}-32 r U_{i-1}^{n+2}+(1+45 r) U_{i}^{n+2}-16 r U_{i+1}^{n+2}+r U_{i+2}^{n+2} \\
& =-30 r U_{i}^{n+1}+32 r U_{i+1}^{n+1}-2 r U_{i+2}^{n+1}-2 r U_{i-2}^{n} \\
& +32 r U_{i-1}^{n}+(1-45 r) U_{i}^{n}+16 r U_{i+1}^{n}-r U_{i+2}^{n} \\
& C_{6}: 2 r U_{i-2}^{n+2}-16 r U_{i-1}^{n+2}+(1+29 r) U_{i}^{n+2}-16 r U_{i+1}^{n+2}+r U_{i+2}^{n+2} \\
& =32 r U_{i-1}^{n+1}-62 r U_{i}^{n+1}+32 r U_{i+1}^{n+1}-2 r U_{i+2}^{n+1}-2 r U_{i-2}^{n} \\
& +16 r U_{i-1}^{n}+(1-29 r) U_{i}^{n}+16 r U_{i+1}^{n}-r U_{i+2}^{n} \\
& C_{7}: r U_{i-2}^{n+2}-16 r U_{i-1}^{n+2}+(1+31 r) U_{i}^{n+2}-16 r U_{i+1}^{n+2} \\
& =-2 r U_{i-2}^{n+1}+32 r U_{i-1}^{n+1}-58 r U_{i}^{n+1}+32 r U_{i+1}^{n+1}-4 r U_{i+2}^{n+1} \\
& -r U_{i-2}^{n}+16 r U_{i-1}^{n}+(1-31 r) U_{i}^{n}+16 r U_{i+1}^{n} \\
& C_{8}: r U_{i-2}^{n+2}-16 r U_{i-1}^{n+2}+(1+15 r) U_{i}^{n+2} \\
& =-2 r U_{i-2}^{n+1}+32 r U_{i-1}^{n+1}-90 r U_{i}^{n+1}+64 r U_{i+1}^{n+1} \\
& -4 r U_{i+2}^{n+1}-r U_{i-2}^{n}+16 r U_{i-1}^{n}+(1-15 r) U_{i}^{n}
\end{aligned}
$$


Thus, it is sufficient to analyze the truncation errors of the formulae $C_{1} \sim C_{8}$. We use $L_{h}^{(1)}-L_{h}^{(8)}$ to represent the discrete operators of $L$ basing on the schemes $C_{1} \sim C_{8}$ respectively, for example, $L_{h}^{(1)}$ can be defined as the following form:

$$
\begin{gathered}
L_{h}^{(1)} u_{i}^{n+1}=\frac{1}{2 \tau}\left\{(1+15 r) u_{i}^{n+2}-16 r u_{i+1}^{n+2}+r u_{i+2}^{n+2}+4 r u_{i-2}^{n+1}-64 r u_{i-1}^{n+1}\right. \\
\left.+90 r u_{i}^{n+1}-32 r u_{i+1}^{n+1}+2 r u_{i+2}^{n+1}-(1-15 r) u_{i}^{n}-16 r u_{i+1}^{n}+r u_{i+2}^{n}\right\}
\end{gathered}
$$

Notice to that the formulae $C_{1} \sim C_{8}$ are all symmetrical schemes with their axis of symmetry $t=t_{n+1}$, then from the Taylor series expansions at the point $\left(x_{i}, t_{n+1}\right)$, we can obtain their truncation errors as below concise forms.

$$
\begin{aligned}
& L_{h}^{(k)} u_{i}^{n+1}-[L u]_{i}^{n+1}=-7 r h \tau\left(\frac{\partial^{3} u}{\partial x \partial t^{2}}\right)_{i}^{n+1}-\frac{7}{12} r h \tau^{3}\left(\frac{\partial^{5} u}{\partial x \partial t^{4}}\right)_{i}^{n+1}+O\left(\tau^{2}+h^{4}+h^{\alpha} \tau^{\beta}\right) \\
& k=1,4 \\
& L_{h}^{(k)} u_{i}^{n+1}-[L u]_{i}^{n+1}=7 r h \tau\left(\frac{\partial^{3} u}{\partial x \partial t^{2}}\right)_{i}^{n+1}+\frac{7}{12} r h \tau^{3}\left(\frac{\partial^{5} u}{\partial x \partial t^{4}}\right)_{i}^{n+1}+O\left(\tau^{2}+h^{4}+h^{\alpha} \tau^{\beta}\right) \\
& k=5,8 \\
& L_{h}^{(k)} u_{i}^{n+1}-[L u]_{i}^{n+1}=r h \tau\left(\frac{\partial^{3} u}{\partial x \partial t^{2}}\right)_{i}^{n+1}+\frac{1}{12} r h \tau^{3}\left(\frac{\partial^{5} u}{\partial x \partial t^{4}}\right)_{i}^{n+1}+O\left(\tau^{2}+h^{4}+h^{\alpha} \tau^{\beta}\right) \\
& k=2,3 \\
& L_{h}^{(k)} u_{i}^{n+1}-[L u]_{i}^{n+1}=-r h \tau\left(\frac{\partial^{3} u}{\partial x \partial t^{2}}\right)_{i}^{n+1}-\frac{1}{12} r h \tau^{3}\left(\frac{\partial^{5} u}{\partial x \partial t^{4}}\right)_{i}^{n+1}+O\left(\tau^{2}+h^{4}+h^{\alpha} \tau^{\beta}\right) \\
& k=6,7 \\
& \text { where } \alpha+\beta=4 .
\end{aligned}
$$

In addition, through further analysis we see also that formulae $C_{1}$ and $C_{5}$ are symmetric about the axis that is the vertical line of $x=$ cons $\tan t$, and the signs of the first two terms of formulae $C_{1}$ and $C_{5}$ of their truncation errors are opposite, and the errors can be eliminated partly. Similarly,

formulae $C_{2}$ and $C_{6}, C_{3}$ and $C_{7}, C_{4}$ and $C_{8}$ have the same property as $C_{1}$ and $C_{5}$. Thus the accuracy of the schemes has been increased.

\section{Numerical Experiments}

We perform the numerical experiments using the following diffusion equation:

$$
\begin{gathered}
\frac{\partial u}{\partial t}=\frac{\partial^{2} u}{\partial x^{2}}, \quad 0<x<2, \quad 0<t<T, \\
u(0, t)=u(2, t), \quad 0<t<T, \\
u(x, 0)=\cos (\pi x), \quad 0<x<2
\end{gathered}
$$

The exact solution is: $u(x, t)=e^{-a \pi^{2} t} \cos (\pi x), 0<x<2$.

Let $u_{i}^{n}=u\left(x_{i}, t_{n}\right)$ and $U_{i}^{n}$ be the exact solution and the approximate solution of the problem respectively, and we calculate the errors in $L_{\infty}-$ norm and $L_{2}-$ norm as: 


$$
E_{\infty, \Delta x}^{n}=\max _{i}\left(e_{\Delta x, \Delta t}^{n}(i)\right)=\max _{i}\left|u_{i}^{n}-U_{i}^{n}\right|, \quad E_{2, \Delta x}^{n}=\left\|e_{\Delta x, \Delta t}^{n}\right\|_{2}=\sqrt{\sum_{i}\left|u_{i}^{n}-U_{i}^{n}\right|^{2} \Delta x},
$$

where $\Delta x$ is the step size in space and $\Delta t$ is the step size in time. The ratios of convergence in space are calculated by

$$
\text { Ratio of convergence } \approx \frac{\log \left(E_{l, \Delta x_{1}} / E_{l, \Delta x_{2}}\right)}{\log \left(\Delta x_{1} / \Delta x_{2}\right)}, l=2, \infty,
$$

when very small time step size $\Delta t$ is taken in computation.

We use a very small time step size $\Delta t=0.1 \times 10^{-5}$ for the three methods in computation of the ratios of convergence in space, the spatial step sizes are taken as $\Delta x=1 / 8,1 / 16,1 / 32,1 / 56$ and $1 / 64$. Table 1 and 2 show results of three methods at the time step number $n=10^{5}$. From the tables, it is clear that the AG- $8 \mathrm{p}$ scheme has a ratio that is nearly fourth order in space, but the AGE [1] and AGE 4-p [8] have a ratio of nearly second order. In terms of errors at different times and mesh paraments, we have compared these numerical methods. Table 3 and 4 give the absolute errors $(a e)$ and the relative errors ( $r e$ ) of numerical solutions. It is obvious that the AG-8p scheme is more accurate than both the AGE method and the AGE 4-p method. From Table 4 and Fig 1, we also see the AG-8p method always has better accuracy than the other two methods for large step size in time $(\lambda=4,2,5)$.

Table 1. The $L_{\infty}$-error and Ratio in Space for the Diffusion Problem

\begin{tabular}{|c|c|c|c|}
\hline \multirow{2}{*}{$\Delta x$} & AG-8p & AGE & AGE-4p \\
\cline { 2 - 4 } & $L_{\infty}-$ error & $L_{\infty}-$ error & $L_{\infty}-$ error \\
& Ratio & Ratio & Ratio \\
\hline $1 / 8$ & $9.5883 e-5$ & $4.7328 e-3$ & $4.7328 e-3$ \\
& -- & -- & -- \\
\hline $1 / 16$ & $6.0540 e-6$ & $1.1822 e-3$ & $1.1822 e-3$ \\
& 3.9853 & 2.0013 & 2.0013 \\
\hline $1 / 32$ & $3.7872 e-7$ & $2.9547 e-4$ & $2.9547 e-4$ \\
& 4.0025 & 2.0002 & 2.0002 \\
\hline $1 / 56$ & $3.8501 e-8$ & $9.6465 e-5$ & $9.6474 e-5$ \\
& 4.1964 & 2.0004 & 2.0001 \\
\hline $1 / 64$ & $2.1151 e-8$ & $7.3850 e-5$ & $7.3861 e-5$ \\
& 4.4858 & 2.0007 & 2.0002 \\
\hline
\end{tabular}

Table 2. The $L_{2}$ - error and Ratio in Space for the Diffusion Problem

\begin{tabular}{|c|c|c|c|}
\hline$\Delta x$ & AG-8p & AGE & AGE-4p \\
\cline { 2 - 4 } & $L_{2}-$ error & $L_{2}-$ error & $L_{2}-$ error \\
Ratio & Ratio & Ratio \\
\hline $1 / 8$ & $9.0624 e-5$ & $4.4732 e-3$ & $4.4732 e-3$ \\
& -- & -- & -- \\
\hline $1 / 16$ & $5.8692 e-6$ & $1.1461 e-3$ & $1.1461 e-3$ \\
& 3.9487 & 1.9646 & 1.9646 \\
\hline $1 / 32$ & $3.7281 e-7$ & $2.9086 e-4$ & $2.9086 e-4$ \\
& 3.9845 & 1.9822 & 1.9822 \\
\hline $1 / 56$ & $3.8149 e-8$ & $9.5603 e-5$ & $9.5611 e-5$ \\
& 4.1872 & 1.9907 & 1.9904 \\
\hline
\end{tabular}




\begin{tabular}{|c|c|c|c|}
\hline $1 / 64$ & $2.0979 e-8$ & $7.3272 e-5$ & $7.3283 e-5$ \\
& 4.4783 & 1.9922 & 1.9918 \\
\hline
\end{tabular}

Table 3. The Comparison of the Numerical Errors for

$$
J=200, \lambda=\tau / h^{2}=0.1, n=1000
$$

\begin{tabular}{|c|c|c|c|c|c|c|c|}
\hline$x(i)$ & $\begin{array}{c}\text { exact } \\
\text { solution } \\
\end{array}$ & \multicolumn{2}{|c|}{ AG-8p } & \multicolumn{2}{|c|}{ AGE-4p[8] } & \multicolumn{2}{|c|}{ AGE[1] } \\
\cline { 3 - 8 } & $(e-7)$ & $\begin{array}{c}\text { re. } \\
(e-7)\end{array}$ & $\begin{array}{c}\text { ae. } \\
(e-6)\end{array}$ & $\begin{array}{c}\text { re. } \\
(e-6)\end{array}$ & $\begin{array}{c}\text { ae. } \\
(e-6)\end{array}$ & $\begin{array}{c}\text { re. } \\
(e-6)\end{array}$ \\
\hline 0.2 & 7.3298 & 0.37199 & 0.50750 & 5.1535 & 7.0309 & 5.7515 & 7.8467 \\
\hline 0.6 & -2.7997 & 1.8675 & 6.6701 & 2.1336 & 7.6205 & 2.2381 & 7.9940 \\
\hline 1.0 & -9.0602 & 1.5261 & 1.6844 & 6.4721 & 7.1435 & 7.1348 & 7.8748 \\
\hline 1.4 & -2.7997 & 0.92425 & 3.3012 & 1.8664 & 6.6665 & 2.1714 & 7.7557 \\
\hline 1.8 & 7.3298 & 2.0974 & 2.8614 & 5.3186 & 7.2561 & 5.7928 & 7.9030 \\
\hline
\end{tabular}

Table 4 The Comparison of the Numerical Errors for

$$
J=400, \lambda=\tau / h^{2}=4, n=1200
$$

\begin{tabular}{|c|c|c|c|c|c|c|c|}
\hline$x(i)$ & \multirow{2}{*}{$\begin{array}{c}\text { exact } \\
\text { solution } \\
\end{array}$} & \multicolumn{2}{|c|}{ AG-8p } & \multicolumn{2}{|c|}{ AGE-4p[8] } & \multicolumn{2}{|c|}{ AGE[1] } \\
\cline { 3 - 8 } & $(e-1)$ & $(e-4)$ & $\begin{array}{c}\text { re. } \\
(e-4)\end{array}$ & $\begin{array}{c}\text { ae. } \\
(e-4)\end{array}$ & $\begin{array}{c}\text { re. } \\
(e-3)\end{array}$ & $\begin{array}{c}\text { ae. } \\
(e-3)\end{array}$ & $\begin{array}{c}\text { re. } \\
(e-3)\end{array}$ \\
\hline 0.2 & 2.4751 & 2.0169 & 8.1487 & 2.8484 & 1.1508 & 1.1594 & 4.6840 \\
\hline 0.6 & -0.94542 & 0.75677 & 8.0046 & 1.0601 & 1.1213 & 0.43165 & 4.5657 \\
\hline 1.0 & -3.0594 & 2.4846 & 8.1212 & 3.5036 & 1.1452 & 1.4261 & 4.6614 \\
\hline 1.4 & 2.4751 & 0.77881 & 8.2377 & 1.1052 & 1.1690 & 0.44975 & 4.7572 \\
\hline 1.8 & -0.94542 & 2.0033 & 8.0937 & 2.8205 & 1.1395 & 1.1482 & 4.6388 \\
\hline
\end{tabular}

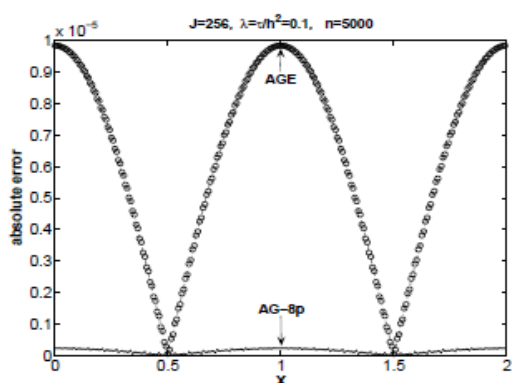

(a) $\lambda=0.1$

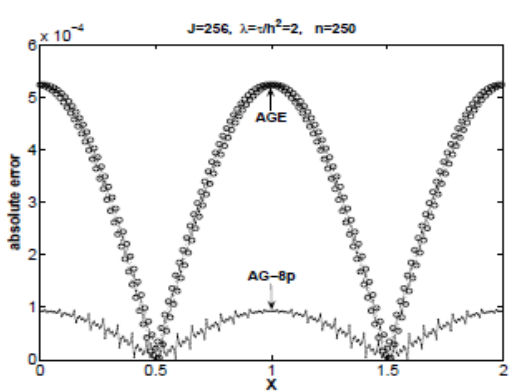

(c) $\lambda=2$

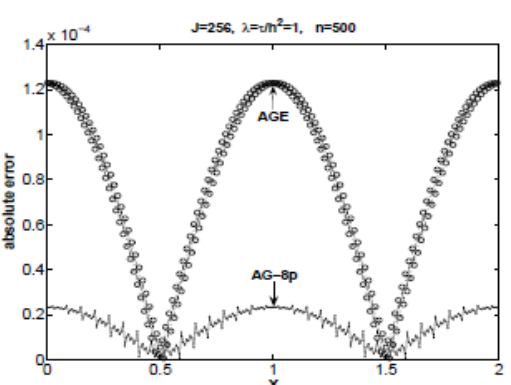

(b) $\lambda=1$

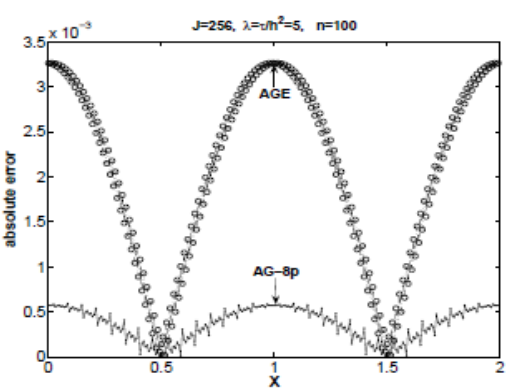

(d) $\lambda=5$

Figure 1. The Comparison of the Absolute Errors at Same Time (Different Numbers of Time Step) and for Different Mesh Paraments $\lambda\left(=\tau / h^{2}\right)$ 


\section{Conclusions}

In this paper, we first construct a group of high-order unsymmetrical schemes $A_{1} \sim A_{8}$ based on the idea of the alternating group method. Next we propose a new alternating group method, whose ratio of convergence is of order 4 in space. The results of our numerical experiments show that the present AG-8p method is efficient and accurate. The method of the AG-8p also can be applied to other partial differential equations.

\section{Acknowledgements}

This paper is supported by the National Natural Science Foundation of China Grant 11271232 .

\section{References}

[1] D. J. Evans and A. R. B. Abdullah, "Group Explicit Methods for Parabolic Equations”, Inter. J. Comp. Math., vol. 14, (1983), pp. 73-105.

[2] D. J. Evans and A. R. B. Abdullah, "A New Explicit Method for the Diffusion-Convection Equation", Comput. Math. Appl. 11, (1985), pp. 145-154.

[3] B. Zhang and W. Li, "On Alternating Segment Crank-Nicolson scheme", Parallel Computing, vol. 20, (1994), pp. 897-902.

[4] Z. Bao-Lin and G. Tong-Xiang, "Principles and Methods of Numerical Parallel Computation", Beijing: National Defence Industry Press, (1999), pp. 205-227.

[5] Z. Zhang and T. Wang, "The Alternating Group Explicit Parallel Algorithm for Convection Dominated Diffusion Problem of Variable Coefficient”, Inter. J. Comp. Math., vol. 81, (2004), pp. 823-834.

[6] Z. Qing-Jie, W. Wen-Qia, "A new alternating group explicit-implicit algorithm with high accuracy for dispersive equation”, Appl. Math. Mech. -Engl. Ed., vol. 29, no. 9, (2008), pp. 1221-1230.

[7] W. Wenqia, "The Alternating Segment Crank-Nicolson Method for Solving Convection-Diffusion Equation", Computing, vol. 73, (2004), pp. 41-55.

[8] W. Wang, "A Class alternating group explicit scheme for solving diffusion equation and parallel computing", Journal of Shandong University, vol. 37, no. 3, (2002), pp. 194-199.

[9] S. Zhu, G. Yuan and L. Shen, "Alternating group explicit method for the dispersive equation”, Intern. J. Comput. Math., vol. 75, (2000), pp. 97-105.

[10] W. Wang and S. Fu, "An unconditionally stable alternating segment difference scheme of eight points for the dispersive equation", Int. J. Numer. Meth. Eng, vol. 67, (2006), pp. 435-447.

[11] C. N. Dawson, Q. Du and T. F. Dupont, "A finite difference domain decomposition algorithm for numerical solution of the heat equation", Mathematical Computation, vol. 57, (1991), pp. 63-71.

[12] G. Yuan, S. Zhu and L. Shen, "Domain decomposition algorithm based on the group explicit formula for the heat equation", Inter. J. Comp. Math., vol. 82, (2005), pp. 1295-1306.

[13] S. Zhu, "A higher-order alternating group explicit scheme for the diffusion equation", Inter. J. Comp. Math., vol. 82, no. 12, (2005), pp. 1497-1503.

[14] R. B. Kellogg, “An Alternating Direction Method for Operator Equation”, SIAM, vol. 12, (1964), pp. 848-854.

\section{Authors}

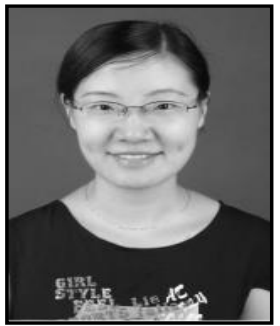

Fuli Qu, received the Bachelor Degree and the Master Degree in computational mathematics from Shandong University, China in 2003 and 2006 respectively. She is a lecturer in Shandong Women's University. Her current research interests on numerical solution of partial differential equation. 


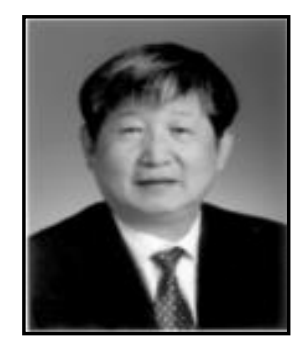

Wenqia Wang, received the Bachelor Degree and the Master Degree in computational mathematics from Shandong University, China in 1974 and 1980 respectively. He is a Professor in Shandong University. His current research interests on numerical solution of partial differential equation and the scientific engineering computing. 